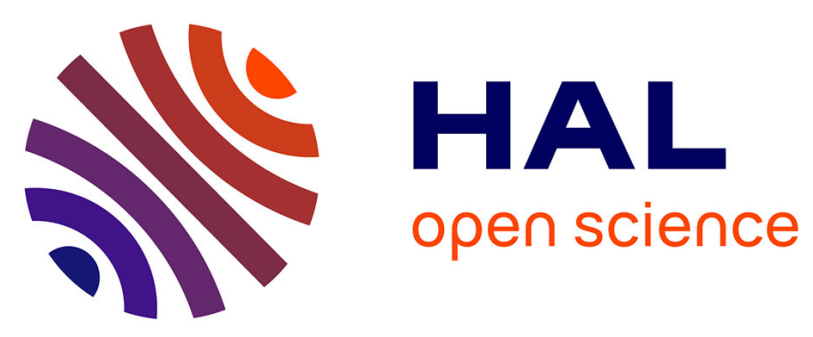

\title{
Appareil pour l'étude des propriétés extensométriques des couches minces - Mesure de variations relatives de résistance entre 10-2 et $5 \times 10-6$ sur des couches dont la résistance peut être comprise entre 300 ohms et 15000 ohms
}

\author{
J.-C. Lecordier
}

\section{To cite this version:}

J.-C. Lecordier. Appareil pour l'étude des propriétés extensométriques des couches minces - Mesure de variations relatives de résistance entre 10-2 et $5 \times 10-6$ sur des couches dont la résistance peut être comprise entre 300 ohms et 15000 ohms. Revue de Physique Appliquée, 1968, 3 (1), pp.1-4. 10.1051/rphysap:01968003010100 . jpa-00242814

HAL Id: jpa-00242814

https://hal.science/jpa-00242814

Submitted on 1 Jan 1968

HAL is a multi-disciplinary open access archive for the deposit and dissemination of scientific research documents, whether they are published or not. The documents may come from teaching and research institutions in France or abroad, or from public or private research centers.
L'archive ouverte pluridisciplinaire HAL, est destinée au dépôt et à la diffusion de documents scientifiques de niveau recherche, publiés ou non, émanant des établissements d'enseignement et de recherche français ou étrangers, des laboratoires publics ou privés. 


\title{
REVUE DE PHYSIQUE APPLIQUÉE
}

\author{
Supplément au "Journal de Physique "
}

\section{APPAREIL POUR L'ÉTUDE DES PROPRIÉTÉS EXTENSOMÉTRIQUES DES GOUGHES MINGES
Mesure de variations relatives de résistance entre $10^{-2}$ et $5 \times 10^{-6}$ sur des couches dont la résistance peut être comprise entre 300 ohms et 15000 ohms

\author{
Par J.-C. LEGORDIER,
}

Laboratoire de Physique des Couches Minces, Faculté des Sciences de Rouen, 76-Mont-Saint-Aignan.

(Reçu le 7 juillet 196\%.)

Résumé. - L'étude des propriétés extensométriques des couches minces métalliques conduit à utiliser des dépôts dont la résistivité varie dans des proportions telles qu'il est impossible d'obtenir des résistances ajustées à une certaine valeur uniquement en leur donnant une géométrie appropriée. Nous avons donc été amené à réaliser un montage permettant de mesurer des variations relatives $\mathrm{d} R / R$ de résistances comprises entre $10^{-2}$ et $5 \times 10^{-6}$, ceci avec une sensibilité indépendante de la valeur de ces résistances dans une plage comprise entre 300 ohms et 15000 ohms.

Abstract. - The device described in this paper was designed to study the performance of thin film strain gages. The relative resistance changes of the strain gage are converted into relative frequency variations through the channel of a Wien bridge oscillator. The strain gage is inserted in an arm of the Wien bridge. After frequency conversion, frequency changes are measured with a differential frequency meter. The measurement of relative resistance variations between $10^{-2}$ and $5 \times 10^{-6}$ are possible. The sensitivity is independant of the value of the gage resistance in the range 300 to $15000 \mathrm{ohms}$.

Le principe de la mesure est le suivant : on transforme directement la variation de résistance en variation de fréquence par l'intermédiaire d'un oscillateur à pont de Wien.

La fréquence d'oscillation d'un tel montage est :

$$
F=\frac{1}{2 \pi \sqrt{R R^{\prime} C C^{\prime}}} \text {. }
$$

Si la résistance $R$ varie de $\mathrm{d} R$, on obtient une variation relative de fréquence :

$$
\frac{\mathrm{d} F}{F}=-\frac{1}{2} \frac{\mathrm{d} R}{R}
$$

Si l'on s'arrange, par variation des valeurs des éléments $R^{\prime} C^{\prime} C$, pour que la fréquence $F$ soit la même pour toutes les valeurs de résistance $R$, la mesure de $\mathrm{d} R / R$ revient donc à une mesure de variation de fréquence :

$$
\mathrm{d} F=-\frac{F}{2} \frac{\mathrm{d} R}{R}
$$

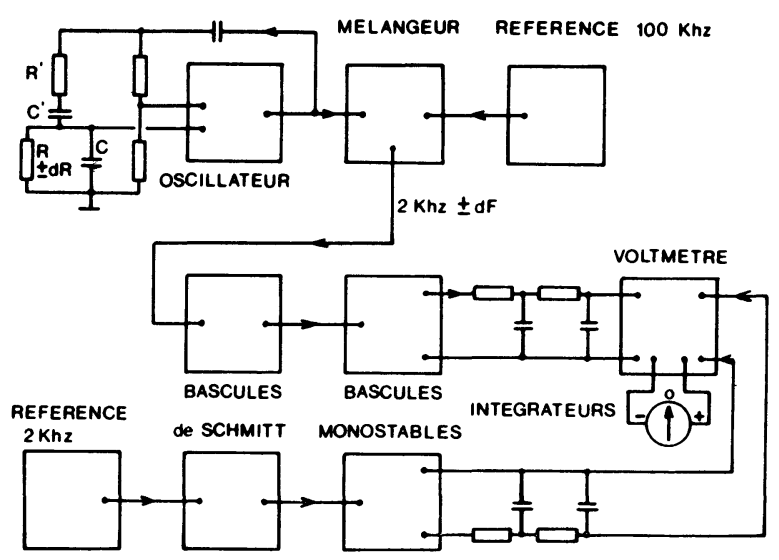

FIG. 1. - Schéma de principe.

Choíx de fréquence d'oscillation. - On voit que les variations $\mathrm{d} F$ de fréquence pour un $\mathrm{d} R / R$ donné sont proportionnelles à la fréquence d'oscillation. On 
aura donc intérêt à prendre $F$ aussi élevé que possible. Il existe cependant une limitation due aux valeurs maxima des résistances de jauge que l'on veut introduire dans le montage; en effet, pour ces valeurs, les capacités $C$ et $C^{\prime}$ devront rester supérieures aux capacités parasites du montage (câbles de liaison, capacité d'entrée du montage).

L'amplificateur doit avoir un gain sans contreréaction important et un déphasage aussi faible que possible à la fréquence d'oscillation, ce qui introduit également une limitation à la valeur de cette fréquence.

En considérant ces différents éléments, la fréquence $F$ a été fixée à $10^{5} \mathrm{~Hz}$.

Pour maintenir la fréquence de l'oscillateur à cette valeur lorsque l'on utilise des jauges de résistance $R$, on ajuste $R^{\prime}$ à la même valeur que celle de la jauge et on ramène la fréquence à la valeur choisie par variation des capacités $C$ et $C^{\prime}\left(C=C^{\prime}\right)$.

Mesure de $\mathrm{d} F$. - La mesure de $\mathrm{d} R / R$ revient donc à la détermination de $\mathrm{d} F$. Étant donné les faibles valeurs de $\mathrm{d} F / F$ que l'on veut pouvoir détecter $\left(10^{-6}\right)$, il est pratiquement impossible de mesurer directement $\mathrm{d} F$.

Pour obtenir $\mathrm{d} F$, on pourrait faire battre la tension de fréquence $F \pm \mathrm{d} F$ avec un signal de fréquence $F$. Ce procédé ne donne pas le signe de $\mathrm{d} F$. On ne connaîtrait donc pas le signe de $\mathrm{d} R$.

Certaines couches minces ayant un coefficient d'extensométrie négatif, la connaissance de ce signe est nécessaire. Pour éviter cet inconvénient, on fait battre les deux oscillateurs à une fréquence $F^{\prime}$ telle que $F$ reste toujours supérieure aux valeurs maxima $\mathrm{de} d F$.

Avec un oscillateur à pont de Wien, une variation relative de résistance de $\pm 10^{-2}$ correspond à une variation de fréquence de $\pm 500 \mathrm{~Hz}$. En prenant pour $F^{\prime}$ la valeur $2000 \mathrm{~Hz}$, on dispose d'une marge de sécurité importante et on élimine en même temps le risque que l'oscillateur $R C$ se synchronise sur l'oscillateur à quartz dans une certaine plage de part et d'autre du battement nul.

On obtient donc, après filtrage à la sortie de l'étage mélangeur, une tension alternative de fréquence $2000 \mathrm{~Hz}$ variant de $\pm \mathrm{d} F$ lorsque $R$ varie de $\mp \mathrm{d} R$.

Oscillateur à pont de Wien. - Cet oscillateur est la partie principale du montage, de sa stabilité dépend en effet toute la précision de la mesure de $\mathrm{d} R / R$.

Dans ce type d'oscillateur, l'instabilité en fréquence est en général due en plus grande part aux variations du déphasage propre de l'amplificateur. Une première condition pour obtenir une bonne stabilité est de rendre ce déphasage indépendant de la fréquence. D'autre part, si l'amplificateur introduit un déphasage, l'oscillation se produira à une fréquence telle que la somme des déphasages de l'amplificateur et du pont de Wien soit nulle. C'est-à-dire à une fréquence différente de celle donnée par :

$$
F=\frac{1}{2 \pi \sqrt{R R^{\prime} C C^{\prime}}}
$$

Dans le cas où l'on veut faire fonctionner l'oscillateur à une fréquence se situant vers la limite supérieure de la bande passante de l'amplificateur, le sens du déphasage est tel que l'oscillation se produit à une fréquence

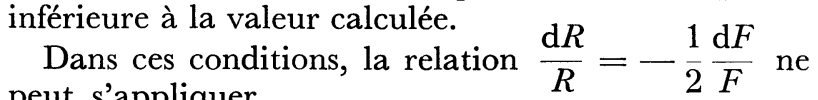
peut s'appliquer.

Or, c'est précisément la vérification de cette expression avec le plus de précision possible qui nous intéressait.

Nous avons donc été amené à corriger la courbe de phase de l'amplificateur de façon à l'annuler dans une plage de fréquence aussi large que possible de part et d'autre de la fréquence d'oscillation choisie : $100 \mathrm{kHz}$ dans notre cas.

Ces exigences nous ont amené à réaliser un amplificateur à deux étages $T_{1} T_{2}$ à large bande suivi par un étage à charge cathodique. Dans cet amplificateur, les résistances de charge ont été réduites à une valeur minimum compatible avec la nécessité de conserver un gain important. Malgré cette précaution, le déphasage global en boucle ouverte de cet amplificateur est de $12^{\circ}$ à $100 \mathrm{kHz}$.

Deux selfs en série avec les charges des tubes $T_{1}$ et $T_{2}$ permettent de ramener à $180^{\circ}$ le déphasage de chaque étage, à $100 \mathrm{kHz}$ ( fig. 2).

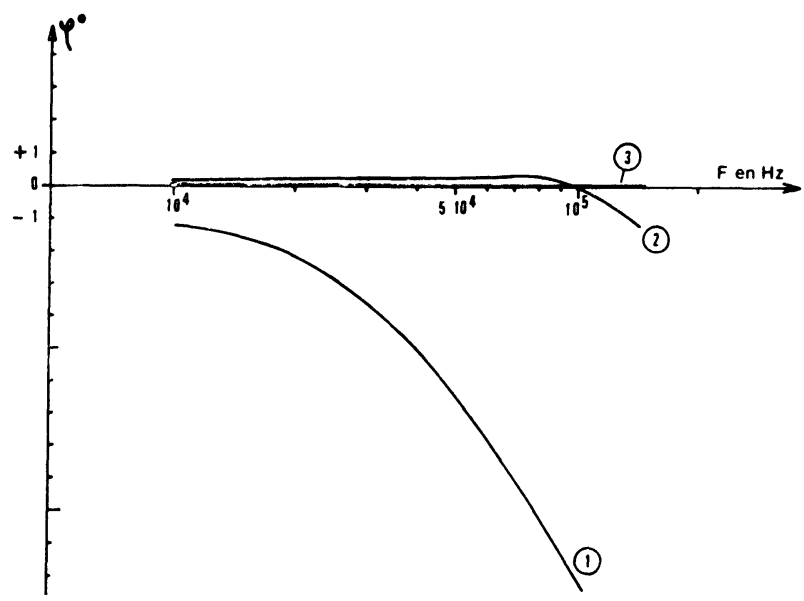

FIG. 2. - Courbe 1 : Déphasage global de l'amplificateur en boucle ouverte. - Courbe 2 : Déphasage global de l'amplificateur en boucle ouverte après correction. Courbe 3 : Déphasage de l'amplificateur corrigé avec contre-réaction correspondant à celle de la thermistance en fonctionnement.

Dans le cas particulier qui nous occupe, on devrait avoir la possibilité d'utiliser dans le pont des jauges de faible résistance de l'ordre de 300 ohms. Il faut donc utiliser dans le pont de Wien des capacités de forte valeur. Pour éviter de charger le dernier étage de l'amplificateur par une impédance faible, nous avons introduit dans la boucle de retour un étage à charge cathodique $\mathrm{T}_{4}$ à faible résistance interne ( fig. 3). 
L'amplitude est limitée à $14 \mathrm{~V}_{\text {eff. }}$ par une thermistance placée dans une des branches du pont de Wien.

En utilisant dans le pont de Wien des éléments $R$ et $C$ de haute stabilité, les résultats obtenus après correction du déphasage sont les suivants pour une fréquence d'oscillation voisine de $100 \mathrm{kHz}$ :

- une variation de $10 \%$ de la tension continue d'alimentation provoque une variation de fréquence de $0,035 \%$;

- une variation de $10 \%$ de la tension alternative de chauffage des tubes provoque une variation de fréquence de $0,017 \%$.

En stabilisant les tensions d'alimentation continue et de chauffage de l'amplificateur, on obtient :

- après une minute de préchauffage et jusqu'à la stabilisation de la fréquence une dérive totale inférieure à $10^{-4}$;

- après vingt minutes de fonctionnement, la dérive en fréquence est inférieure à $10^{-6} /$ minute;

- la dérive calculée pendant la deuxième heure de fonctionnement est inférieure à $2 \times 10^{-7} /$ minute.

Nous avons vu qu'il fallait faire battre l'oscillateur à pont de Wien avec un autre oscillateur présentant avec lui un écart de fréquence de $2 \mathrm{kHz}$. Nous avons donc pris pour cela un oscillateur de référence à quartz fonctionnant à $100 \mathrm{kHz}$. Un étage mélangeur classique $T_{5}$ permet d'obtenir le battement à $2000 \mathrm{~Hz} \pm \mathrm{d} F$.

L'oscillateur à pont de Wien sera donc réglé à 98 ou $102 \mathrm{kHz}$ pour faire le zéro de l'appareil.

On peut déterminer sur quel battement on se trouve lorsque l'on a fait le zéro. Il suffit de faire varier dans un sens connu un des éléments du pont de Wien, $C, R^{\prime}$ ou $C^{\prime}$. Le sens de variation de la fréquence du battement permet de savoir si l'oscillateur fonctionne à 98 ou $102 \mathrm{kHz}$.

Fréquencemètre ( fig. 1 et 3 ). - L'étage mélangeur fournit donc une tension alternative de fréquence $2000 \mathrm{~Hz} \pm \mathrm{d} F$. La partie fréquencemètre du montage doit donc nous donner $\mathrm{d} F$ avec son signe. Il faut également prévoir un changement de sensibilité simple pouvant être effectué en cours de mesure dans le cas où la variation de fréquence $\mathrm{d} F$ qui apparaît est supérieure à la gamme initiale choisie.

Pour cela, nous avons réalisé un fréquencemètre entièrement symétrique à deux entrées. Sur une des entrées, on applique une tension à $2000 \mathrm{~Hz}$ fournie par un oscillateur de référence. Sur l'autre, celle dont on veut mesurer l'écart de fréquence par rapport à $2 \mathrm{kHz}$.

Schéma : Le signal alternatif est appliqué sur une bascule de Schmitt. Le signal rectangulaire à même fréquence, obtenu à la sortie, est différencié et détecté. L'impulsion négative de $30 \mathrm{~V}$ crête à crête obtenue sert à déclencher une bascule monostable délivrant des impulsions calibrées de $90 \mathrm{~V}$ crête à crête et de $160 \mu$ s de largeur dont la fréquence est égale à celle du signal d'entrée. Ces impulsions sont ensuite intégrées par deux circuits $R C$. On obtient donc à la sortie une tension continue proportionnelle à la fréquence du signal d'entrée.

La tension continue qui apparaît à la sortie de l'autre moitié du montage est identique à la première si on applique à l'entrée une tension alternative de même fréquence. S'il existe une différence de fréquence $\mathrm{d} F$ entre les signaux appliqués sur les entrées, il existe entre les deux tensions continues une différence $\mathrm{d} V$ proportionnelle à $\mathrm{d} F$.

La partie centrale du fréquencemètre est un voltmètre continu permettant d'adapter les impédances des intégrateurs et du galvanomètre sur lequel on veut faire apparaître le signal.

Les différentes gammes sont obtenues en commutant des résistances en série avec le galvanomètre.

Gertains réglages sont nécessaires avant d'utiliser la partie fréquencemètre du montage. Un commutateur à 4 positions permet d'isoler les différentes parties du montage où un réglage s'impose :

1) en position (1) du commutateur : le voltmètre central est isolé, on fait le zéro avec le potentiomètre $\mathrm{P}_{1}$;

2) en position (2) du commutateur : le voltmètre central est relié aux bascules monostables. Il n'y a aucun signal alternatif sur les anodes des tubes $T_{\mathbf{1 4}}$, $T_{17}$, le potentiomètre $P_{3}$ permet d'égaliser les tensions continues d'anode des tubes précédents;

3) la tension de fréquence $2000 \mathrm{~Hz}$ fournie par l'oscillateur de référence est appliquée simultanément sur les deux entrées. Le potentiomètre $\mathrm{P}_{2}$ permet d'égaliser la surface des impulsions fournies par les bascules monostables en ramenant le galvanomètre central à zéro;

4) la position (4) sert à la mesure. Le $2000 \mathrm{~Hz}$ de référence est appliqué sur une seule entrée.

Les résultats obtenus avec ce fréquencemètre sont les suivants :

- les défauts de linéarité apparaissent aux fréquences suivantes : $100 \mathrm{~Hz}$ et $3400 \mathrm{~Hz}$; à ces deux fréquences, l'écart entre la fréquence lue et la fréquence appliquée est de l'ordre de $1 \%$;

- pour des variations relatives de résistance de $\pm 10^{-2}$, la fréquence à l'entrée de cette partie du montage variera entre $1500 \mathrm{~Hz}$ et $2500 \mathrm{~Hz}$.

La précision de la mesure dépendra donc en fait de la précision de l'étalonnage et des appareils de lecture utilisés (galvanomètre, enregistreur, etc.) :

- en variations relatives de résistance, avec une même tension alternative de fréquence $2000 \mathrm{~Hz}$ appliquée sur chaque entrée, le bruit de fond de cet appareil est inférieur à $3 \times 10^{-6}$. 


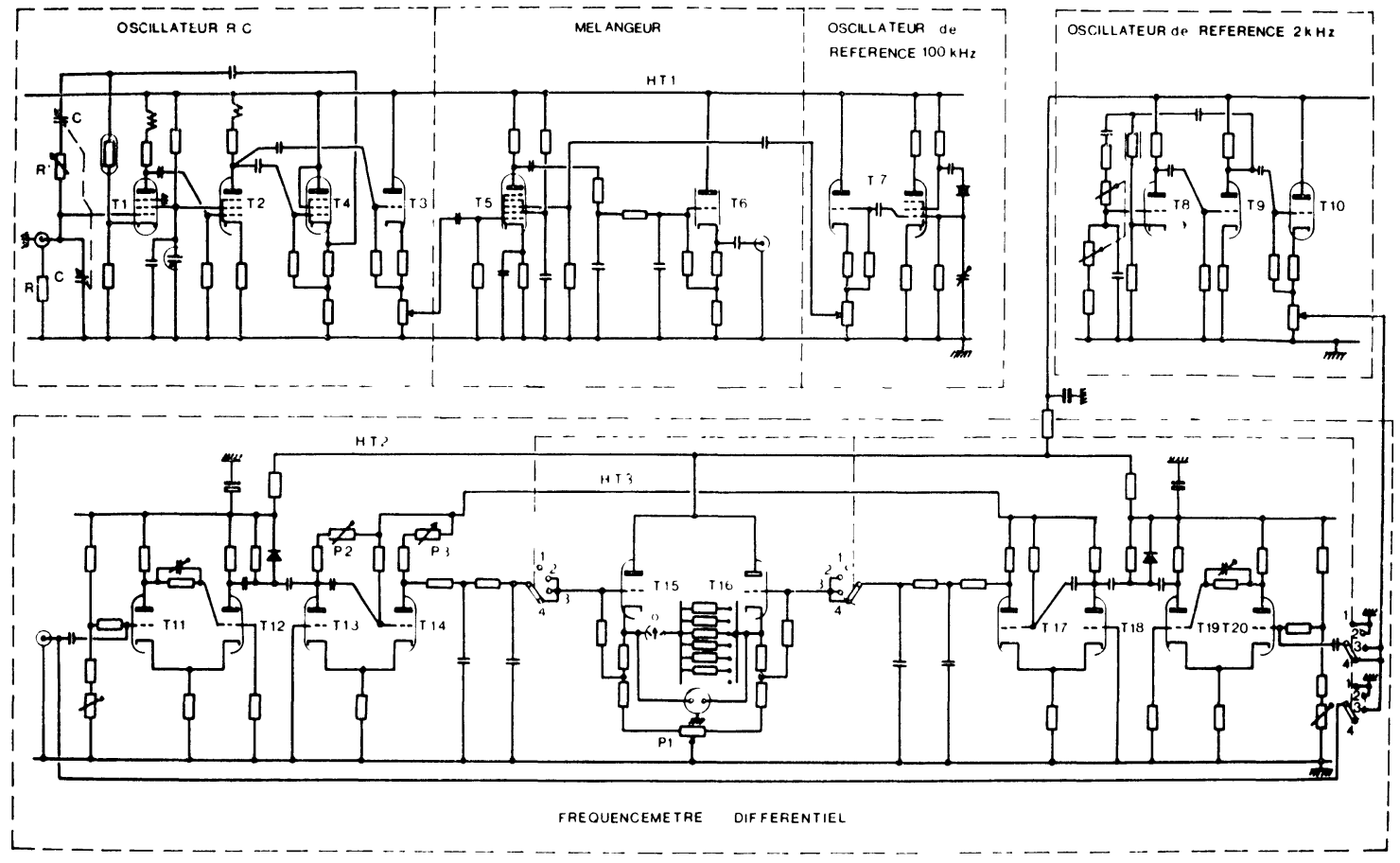

FIG. 3. - Schéma général du dispositif.

Conciusion. - L'ensemble ainsi réalisé permet donc la détection de variations relatives de résistance de l'ordre de $5 \times 10^{-6}$. Les variations relatives de résistance qui apparaissent dans l'étude extensométrique des couches minces sont donc facilement mesurables par ce procédé avec une précision satisfaisante.

Manuscrit reçu le 7 juillet 1967 .

\section{BIBLIOGRAPHIE}

- Electronic Designer's Handbook, Landee, Donovan, Albrecht, McGraw-Hill Book Company.

- Semiconductor and conventional strain gages, Dean, Douglas, Academic Press, N.Y.

- Vacuum tubes and semiconductors Electronics, Millman, McGraw-Hill Book Company.
- MAAS (G.), Instrum. et Lab., $4^{\mathrm{e}}$ trimestre 1957, 2 ; Mesures, déc. 1956, 235 ; Mesures, mars 1957, 238 ; Mesures, septembre 1957, 244.

- A.T.M., Arch. Tech. Messen, juin 1955.

- A.T.M., Arch. Tech. Messen, février 1955. 